\title{
Utilization of dietary purines and pyrimidines by non-ruminant animals
}

\author{
By J. P. F. D'MeLlo, Department of Agricultural Biochemistry, The Edinburgh \\ School of Agriculture, West Mains Road, Edinburgh $\mathrm{EH}_{9}{ }_{3} \mathcal{F} G$
}

\section{Introduction}

Interest in the nutritional significance of dietary nucleic acids and their constituent purine and pyrimidine bases dates back to 1937 when Frost \& Elvehjem (1937) demonstrated the beneficial properties of exogenous nucleotides given to rats fed on low-niacin diets. The early work formed part of the search for unidentified growth factors (UGF) in various dietary ingredients including fish solubles, whey and yeast (Barnett et al. 1956; Eyssen, 1962).

With the advent of large-scale production of single cell protein (SCP) in recent years, interest in nucleic acid metabolism has been revived by claims that the purine and pyrimidine nucleotides in SCP may be salvaged and re-utilized for growth and nitrogen retention by non-ruminant animals. There is some evidence that small SCP inclusions in pig and poultry diets improve $\mathrm{N}$ retention (D'Mello \& Acamovic, 1976; D'Mello et al. 1976) and this effect is occasionally attributed to the utilization of the nucleic acid fraction (Whittemore et al. 1978). On the other hand, there have been claims that nucleic acids from SCP are almost completely catabolized by the pig and, therefore, not retained for tissue synthesis (Newport \& Keal, I980). This appears to be an opportune moment to review the metabolism of nucleic acids in non-ruminant animals.

\section{Synthesis of purine and pyrimidine nucleotides}

Purine and pyrimidine nucleotides may be synthesized de novo from amino acids and other molecules (Fig. I). The de novo pathways are the major routes for the synthesis of the ribonucleotides in animal tissues. While many of the starting materials are common to both pathways, the detailed reactions are completely different. For example, in the de novo synthesis of purines, 5-phosphoribosyl-1pyrophosphate (PRPP) is incorporated in the very first reaction. On the other hand, in the de novo synthesis of pyrimidines, PRPP is incorporated in the terminal stages, after the formation of the pyrimidine ring.

Although the significance of the de novo pathways was recognized many years ago, it was not appreciated until recently that animal cells, in common with other cells, could utilize the free bases and their corresponding nucleosides. These reutilization or salvage pathways are also illustrated in Fig. I. The preformed bases and ribonucleosides may be derived from the diet or from the cellular environment.

It is not often appreciated that some animal cells have an absolute requirement for preformed purines and pyrimidines or their nucleosides owing to a deficiency of one or more of the enzymes in the de novo pathway of synthesis. Thus Miyazaki et al. (1978) concluded that preformed adenosine, arising either from the diet or from 


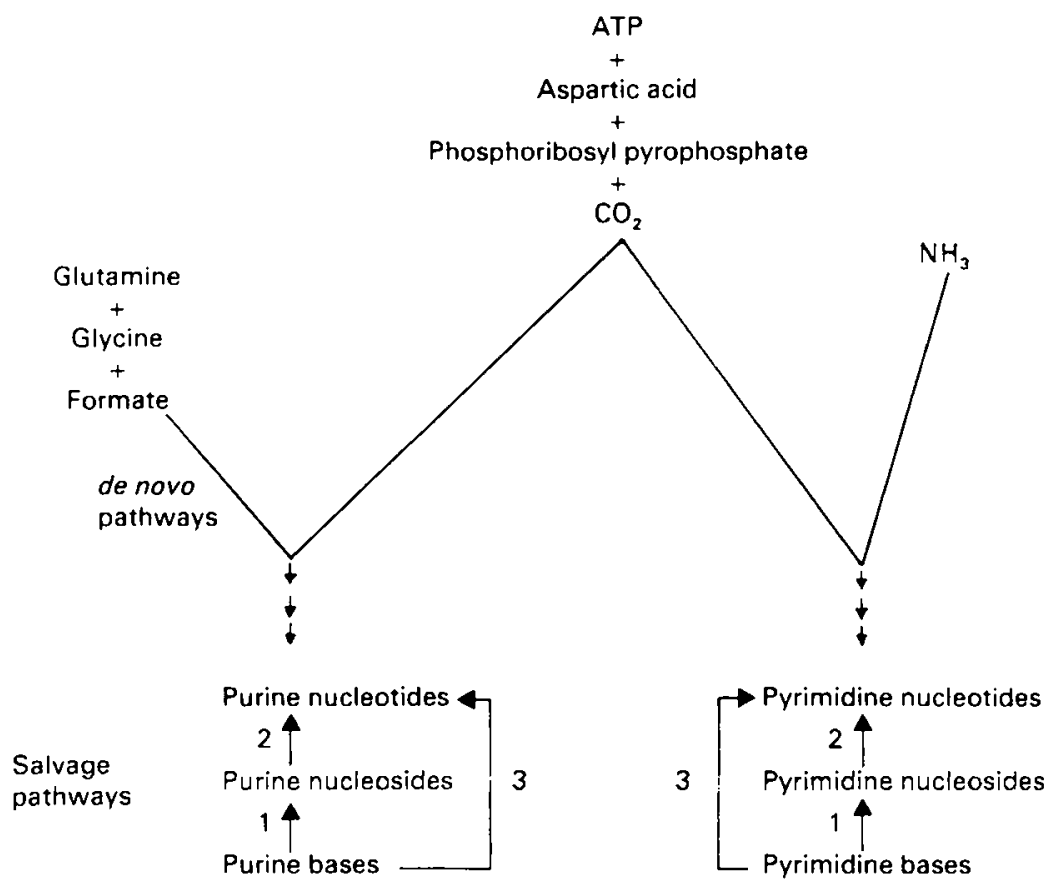

Fig. I. Biosynthesis of purine and pyrimidine nucleotides. Enzymes: 1, nucleoside phosphorylase; 2, nucleoside kinase; 3 , phosphoribosyltransferase.

normal nucleic acid and nucleotide turnover, is utilized by erythrocytes in order to overcome their inability to synthesize purines de novo. Henderson \& Paterson (1973) suggest that many cells, including most animal cells, satisfy part of their purine requirements by synthesis de novo and part through the utilization of preformed purines. It is conceivable that pyrimidine requirements, at the cellular level at any rate, are met in a similar manner. The utilization of preformed pyrimidines by animal cells has not been thoroughly investigated and, consequently, standard works on the biochemistry of the nucleic acids (Davidson, r 969 ) tend to ignore this topic.

The existence of the salvage pathways for purine and pyrimidine re-utilization has been established over a number of years by the use of ${ }^{15} \mathrm{~N}$ - or ${ }^{14} \mathrm{C}$-labelled precursors. Although early investigators were unable to demonstrate utilization due, presumably, to limitations in methodology (see Rose (1923)), the studies of Brown et al. (1948), and those of subsequent investigators (Burridge et al. 1976; Savaiano \& Clifford, 1978), showed conclusively that dietary purines, especially adenine, could be incorporated into tissue nucleic acids. Thus Brown et al. (1948) showed that $14 \%$ of adenine and $8 \%$ of guanine in tissue nucleic acids of rats were derived from dietary adenine administered at a daily rate of $200 \mathrm{mg} / \mathrm{kg}$ bodyweight. Utilization of preformed pyrimidines is less well documented but it seems clear from in vitro (Canellakis, 1957) and in vivo (Hammarsten et al. 1950) studies 
that uracil and cytidine can be utilized by the rat for the synthesis of new nucleotides. For example, when a small dose of ${ }^{15} \mathrm{~N}$-cytidine was injected into rats, cytosine in both RNA and DNA of tissues became more strongly labelled than the other pyrimidines- evidence, perhaps, of a direct route of utilization.

The pattern of tissue retention of preformed bases and nucleosides is fairly well established. The findings of several investigators (Burridge et al. 1976; Sonoda \& Tatibana, 1978; Ho et al. 1979) show that the gastrointestinal tract, liver and skeletal muscle play a major role in the re-utilization of the administered purine or pyrimidine. The involvement of the gut and hepatic tissues as the major sites of reutilization of exogenous bases and nucleosides is perhaps consistent with the relatively high turnover rates of RNA in these tissues. It has been suggested that the intestinal tissues and liver remove exogenous nucleosides and bases by degrading or re-utilizing these components with the consequence that only limited quantities become available for incorporation in other organs such as the kidney, spleen and heart (Ho et al. 1979).

\section{Factors affecting utilization of preformed bases and nucleosides}

The extent to which preformed bases and nucleosides may be salvaged and reutilized for synthesis of tissue nucleotides and nucleic acids is dependent upon a variety of factors.

Type of base. The results of several studies indicate that adenine is more extensively incorporated into the tissues of the mouse and the rat than any other purine base, including guanine (Burridge et al. 1976; Savaiano \& Clifford, 1978; Ho et al. 1979). Xanthine is poorly utilized by the rat (Ho et al. 1979) and uric acid is not utilized at all by the mouse (Miyazaki et al. 1978) or the young chick (Baker \& Molitoris, 1974).

The unique role of adenine in purine salvage and its relationship with guanine deserves special comment. Besides being more efficiently utilized, dietary adenine is readily converted into guanine (Brown et al. 1948) but the existence of the reverse reaction has been much more difficult to establish. Nevertheless, it is probably true to say that adenine and guanine are interconvertible and that this interconversion occurs at the nucleotide level (Fig. 2) with inosinate (IMP) as the central intermediate (Henderson \& Paterson, 1973). The arrangement of these reactions in two separate cycles, with IMP as the common nucleotide, suggests that the interconversion between adenylate (AMP) and guanylate (GMP) should be freely reversible. However, in reviewing the evidence, Henderson \& Paterson (1973) commented on the relatively low activities of guanylate reductase in many animal cells. This might account for the comparatively poor utilization of preformed guanosine and GMP as precursors for the synthesis of AMP.

The superior utilization of preformed adenine, relative to guanine, for tissue nucleotide synthesis may also be explained on the basis that the evidence for the existence of adenine deaminase in animal cells is, at best, insubstantial. Indeed, Henderson \& Paterson (1973) believe this enzyme to be totally absent in animal 


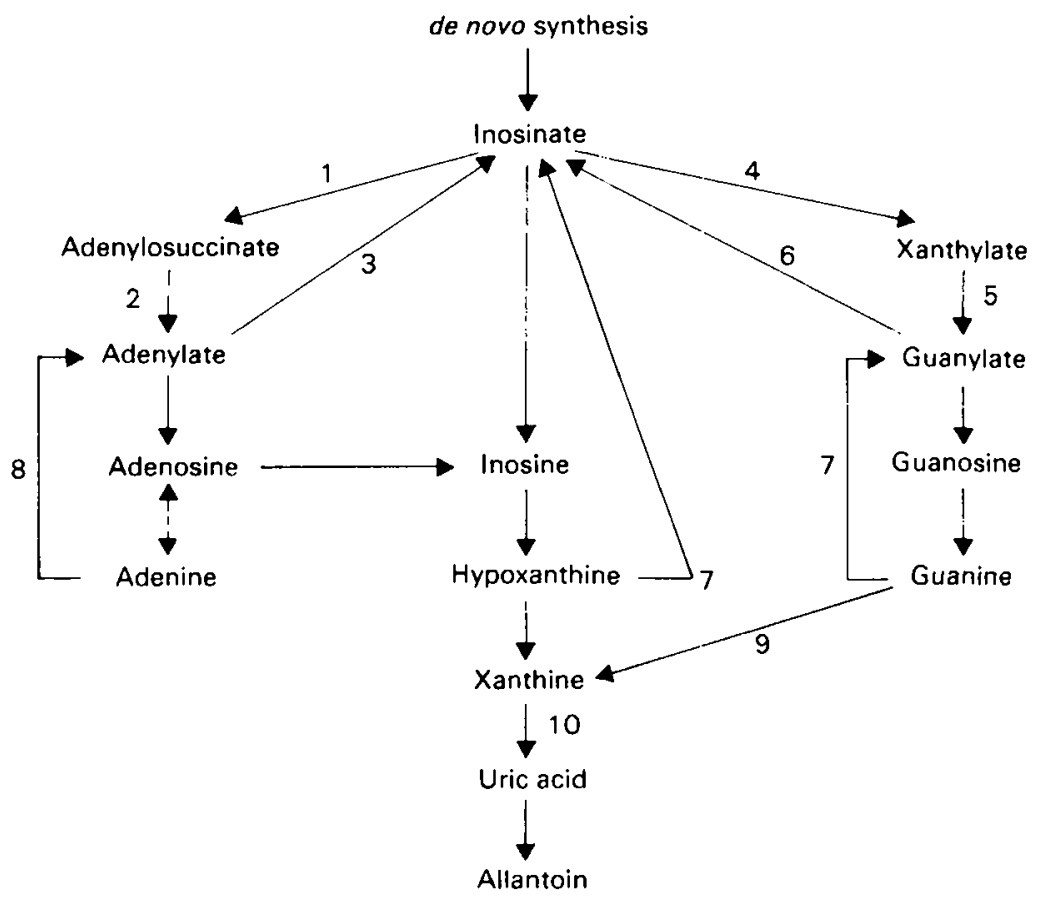

Fig. 2. Interconversion, salvage and catabolism of purine nucleotides in animal tissues. Enzymes: I, adenylosuccinate synthetase; 2 , adenylosuccinate lyase; 3 , adenylate deaminase; 4 , inosinate dehydrogenase; 5 , guanylate synthetase; 6 , guanylate reductase; 7 , hypoxanthine-guanine phosphoribosyltransferase; 8 , adenine phosphoribosyltransferase; 9 , guanine deaminase; 10, xanthine oxidase.

tissues. This suggests that, in view of the doubtful role of purine nucleoside phosphorylase in converting adenine to adenosine, an important metabolic route for dietary adenine is essentially one of salvage via phosphoribosyltransferase. The results of Clifford \& Story (1976) tend to support this view (Table I). The addition of adenine to a control diet induced significant increases in the hepatic activities of adenine phosphoribosyltransferase, $5^{\prime}$-nucleotidase and adenosine deaminase in the rat. On the other hand, urinary excretion of the purine was unaffected by adenine addition.

Another reason for the preferential utilization of dietary adenine centres on the apparent ability of some animals to absorb adenine from the intestine without any alteration to the molecule, whereas guanine and other purines do not cross the mucosal wall as free bases (Savaiano et al. $\mathrm{r} 980$ ).

The inferior utilization of guanine is attributable not only to poor intestinal absorption of the free base and low activities of guanylate reductase but also to the existence of an active guanine deaminase in most animal cells (Henderson \& Paterson, 1973). This provides a direct route for the catabolism of guanine to uric acid and/or allantoin (Fig. 2). These comments probably also help to explain the inability of the pig to retain guanine (Simmonds et al. 1973). 
Table I. Effect of dietary adenine on hepatic enzyme activities and urinary purine excretion in the rat*

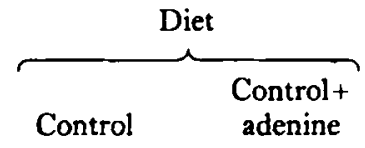

Hepatic enzyme activity ( $\mu \mathrm{M}$ of product formed/g liver post-microsomal extract protein per $\mathrm{h}$ )

$\begin{array}{lll}\text { Adenine phosphoribosyltransferase } & 120 & 187 \\ 5^{\prime}-\text { Nucleotidase } & 191 & 267 \\ \text { Adenosine deaminase } & 401 & 619 \\ \text { inary purine excretion }(\mu \mathrm{M} / \mathrm{rod}) & & \\ \text { Guanine } & 7 \cdot 1 & 8 \cdot 1 \\ \text { Adenine } & 2 \cdot 5 & 1 \cdot 5\end{array}$

-Adapted from Clifford \& Story (1976).

Table 2. Recovery of radioactivity from liver, carcass and urine of fed and fasted rats given a single intravenous or oral dose of $\left[8-{ }^{14} \mathrm{C}\right]$ adenine (proportion of total radioactivity)*

$\begin{array}{ccccc} & \overbrace{\text { Fed }} & \text { Fasted } & \overbrace{\text { Fed }} & \text { Fasted } \\ \text { Liver } & 0.055 & 0.046 & 0.004 & 0.100 \\ \text { Carcass } & 0.416 & 0.394 & 0.020 & 0.036 \\ \text { Urine } & 0.249 & 0.285 & 0.938 & 0.861 \\ & \text { *Adapted from Savaiano et al. (1980). }\end{array}$

With regard to the pyrimidines, there appears to be little information on the relative potency of uracil, cytosine and thymine, although it has been reported that thymine is toxic to young chicks (D'Mello, I979).

Method of administration. Although the intestinal absorption of dietary adenine proceeds rapidly, the tissue retention of this purine occurs more efficiently when it is administered by intravenous injection. The effect of intravenous administration of adenine is most clearly seen in the work of Savaiano et al. (1980) which is reproduced in part in Table 2 . The injection of a single dose of $\left[8-{ }^{14} \mathrm{C}\right]$ adenine into the tail vein of rats resulted in extensive incorporation into the carcass and decreased output in the urine of the administered label, in comparison with an identical oral dose of the same labelled purine.

It is probable that absorbed purines, after transportation to the liver, are directed towards a predominantly catabolic route. The intravenous route of administration, on the other hand, in by-passing the liver directs purines towards peripheral tissues engaged in predominantly anabolic activities.

Intravenous injections do not always result in enhanced tissue incorporation of the administered purine, as shown by the results of Miyazaki et al. (1978). These 
investigators used a whole body autoradiographic technique for the assessment of the distribution of labelled purines in the mouse. Mice given intravenous injections of hypoxanthine catabolized virtually all the purine within $8 \mathrm{~h}$ of administration. However, the fate of hypoxanthine administered in this way is altered substantially by pretreatment of the animals with allopurinol. This compound is similar in structure to hypoxanthine and is a potent inhibitor of xanthine oxidase. It is sometimes used clinically in the treatment of gout. The treatment of mice with allopurinol, prior to intravenous administration of labelled hypoxanthine, resulted in considerable retention of hypoxanthine which was presumably transformed into tissue nucleotides (Miyazaki et al. 1978).

Broadly similar results have been obtained with the pig (Simmonds et al. 1973). In this case, allopurinol treatment prior to intravenous administration of labelled guanine, resulted in rapid incorporation of this purine into body tissues with a slow subsequent daily excretion of the label in faeces and urine.

Taken as a whole, these results suggest that after allopurinol-induced inhibition of purine catabolism, the salvage pathways involving hypoxanthine and guanine phosphoribosyltransferases become activated, leading to the conservation of the administered purines.

Nutritional status. The results in Table 2 (Savaiano et al. 1980) illustrate the effect of an overnight fast on adenine retention by the rat. The oral administration of adenine resulted in enhanced hepatic incorporation of the purine by fasted rats in comparison with fed rats. This difference between fed and fasted animals did not occur after intravenous administration of the same dose of adenine. The authors proposed a complementary relationship between de novo purine synthesis, which is dominant in the fed state, and purine salvage, which becomes dominant during fasting when the availability of precursors is restricted.

Dietary factors. It is well established that the use of purified diets provides ideal conditions for the demonstration of purine and pyrimidine utilization. Some of the results referred to in this paper have been obtained by the use of purified, purinefree diets (Clifford \& Story, 1976 ).

Among the dietary factors, $\mathrm{N}$ content has perhaps the most significant effect on the utilization of preformed purines and pyrimidines. This has been demonstrated with young chicks fed on semi-purified $\mathrm{N}$-limiting diets (Table 3 ). The diets were adequately supplemented with the complete range of indispensable amino acids. The basal diet was supplemented with adenine, uracil, adenine + uracil or glutamic acid. Each supplement or combination of supplements provided an additional $2.33 \mathrm{~g} \mathrm{~N} / \mathrm{kg}$ diet. Supplemented diets were, therefore, isonitrogenous. Adenine or uracil additions had no effect on growth or food utilization, although efficiency of $\mathrm{N}$ deposition in the carcass was reduced significantly. Combined additions of adenine and uracil, on the other hand, substantially improved growth to levels near those attained by chicks receiving glutamic acid. Birds in the latter group utilized dietary $\mathrm{N}$ more efficiently than those given adenine with uracil.

When dietary $\mathrm{N}$ concentrations were increased slightly, but still maintained at sub-optimal levels (Table 4 ), adenine + uracil additions were less effective in 
Table 3. Daily weight gain (DWG, g/chick), efficiency of food conversion (EFC, $g$ gain/g DM consumed) and efficiency of $N$ deposition in carcass (END, $g N$ deposited/g $N$ consumed) of chicks fed $N$-limiting diets* supplemented with adenine, uracil or L-glutamic acid $\dagger$

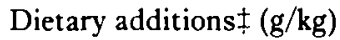

None (basal, only)

+ Adenine (4.5)

+ Uracil $(9 \cdot 3)$

+ Adenine $(2 \cdot 2)+\operatorname{uracil}(4 \cdot 8)$

+ Glutamic acid (24.5)

$\begin{array}{ccc}\text { DWG } & \text { EFC } & \text { END } \\ 11.6 & 0.490 & 0.633 \\ 11.1 & 0.487 & 0.589 \\ 11.8 & 0.487 & 0.561 \\ 13.6 & 0.520 & 0.605 \\ 14.6 & 0.550 & 0.660\end{array}$

* $\mathrm{N}$ content, $19.84 \mathrm{~g} / \mathrm{kg} \mathrm{DM}$, adequately supplemented with all indispensable amino acids. Ingredients $(\mathrm{g} / \mathrm{kg})$ : maize, 754 ; sucrose, 85.5 ; maize oil, 50 ; mineral and vitamins, 56; indispensable amino acid mixture, $54 \cdot 5$.

$\because$ Adapted from D'Mello (1979).

tEach supplement or combination of supplements provided an additional $2 \cdot 33 \mathrm{~g} \mathrm{~N} / \mathrm{kg}$ diet. Supplemented diets were, therefore, isonitrogenous.

Table 4. Daily weight gain ( $D W G, g /$ chick), efficiency of food conversion $(E F C, g$ gain $/ g$ DM consumed) and efficiency of $N$ deposition in carcass (END, $g N$ deposited/g $N$ consumed) of chicks fed N-limiting diets" supplemented with selected purines, pyrimidines or $\mathrm{L}-$ glutamic acid $†$

Dietary additions $+\frac{\ddagger}{+}(\mathrm{g} / \mathrm{kg})$

None (basal only)

+ Uracil (4.8) + Adenine (2.3)

+ Uracil (2:4) + Adenine (I-I) + Guanine (I 3$)$

+ Cytosine (1.5)

+ Glutamic acid $(24 \cdot 5)$

$\begin{array}{ccc}\text { DWG } & \text { EFC } & \text { END } \\ 19.4 & 0.608 & 0.663 \\ 20.2 & 0.625 & 0.608 \\ & & \\ 22.0 & 0.639 & 0.651 \\ 22.6 & 0.665 & 0.685\end{array}$

* $\mathrm{N}$ content, $23.36 \mathrm{~g} / \mathrm{kg} \mathrm{D} . \mathrm{r}$, adequately supplemented with all indispensable amino acids. Ingredients $(\mathrm{g} / \mathrm{kg})$ : maize, 693; sucrose, 107.4 ; maize oil, 36.5 ; minerals and vitamins, 43.5 ; soya-bean, 90 ; indispensable amino acid mixture, $29 \cdot 6$.

$\dagger$ Adapted from D'Mello (1979).

$t$ Each supplement or combination of supplements provided an additional $2 \cdot 33 \mathrm{~g} \mathrm{~N} / \mathrm{kg} \mathrm{diet;}$ supplemented diets were, therefore, isonitrogenous.

promoting growth, but supplements of adenine + uracil + guanine + cytosine appeared to be highly effective. As in the previous experiment (Table 3), however, glutamic acid- $\mathrm{N}$ was utilized more efficiently than purine- and pyrimidine- $\mathrm{N}$.

These responses suggest that both dietary $\mathrm{N}$ content and base composition of the mixture are important factors governing purine and pyrimidine utilization. Although the results suggest salvage and re-utilization, it is possible that these bases were being used as $\mathrm{N}$ sources for the manufacture of the dispensable amino acids. In this respect, it is appropriate to recall the work of Baker $\&$ Molitoris (1974) who showed that chicks fed purified dicts totally devoid of the dispensable amino acids, responded positively to individual dietary supplements of either uracil or adenine. 


\section{Conclusions}

The salvage of preformed purines and pyrimidines occurs widely in nonruminant animals. However, it should be stressed that extensive catabolism of exogenous bases also occurs in these animals. The effect of dietary purines and pyrimidines is to enhance activities of salvage and catabolic enzymes and to suppress de novo synthesis of nucleotides (Leleiko et al. 1979). Therefore, it seems reasonable to conclude that any tissue supplied with exogenous bases will conserve resources by switching off de novo synthesis.

\section{REFERENCES}

Baker, D. H. \& Molitoris, B. A. (1974). f. Nutr. 104, 553.

Barnett, B. D., Lapidus, M., Bird, H. R. \& Strong, F. M. (1956). Proc. Soc. exp. Biol. Med. 92, 372.

Brown, G. B., Roll, P. M., Plentl, A. A. \& Cavalieri, L. F. (1948). F. biol. Chem. 172, 469.

Burridge, P. W., Woods, R. A. \& Henderson, J. F. (1976). Can. F. Biochem. 54, 500.

Canellakis, E. S. (1957). F. biol. Chem. 227, 701.

Clifford, A. J. \& Story, D. L. (1976). F. Nutr. 106, 435.

Davidson, J. N. (1969). The Biochemistry of the Nucleic Acids, 6th ed., London: Methuen \& Co. Ltd.

D'Mello, J. P. F. (1979). F. Sci. Fd Agric. 30, $3^{81}$.

D'Mello, J. P. F. \& Acamovic, T. (1 976). Br. Poult. Sci. 17, 393.

D'Mello, J. P. F., Peers, D. G. \& Whittemore, C. T. (1976). Br. F. Nutr. 36, 403.

Eyssen, H. (1962). Poult. Sci. 41, 1822.

Frost, D. V. \& Elvehjem, C. A. (1937). J. biol. Chem. $121,255$.

Hammarsten, E., Reichard, P. \& Saluste, E. (1950). F. biol. Chem. 183, 105.

Henderson, J. F. \& Paterson, A. R. P. (1973). Nucleotide Metabolism, an Introduction. London: Academic Press.

Ho, C. Y., Miller, K. V., Savaiano, D. A., Crane, R. T., Ericson, K. A. \& Clifford, A. J. (1979). J. Nutr. 109, 1377 .

Leleiko, N. S., Bronstein, A. \& Munro, H. N. (1979). Pediatric Res. 13, 403.

Miyazaki, H., Matsunaga, Y. \& Hashimoto, M. (1 978). F. Histochem. Cytochem. 26, 663.

Newport, M. J. \& Keal, H. D. (1980). Br. F. Nutr. 44, I6I.

Rose, W. C. (1923). Physiol. Rev. 3, 544.

Savaiano, D. A. \& Clifford, A. J. (1978). Nutr. Rep. Int. 17, 55 I.

Savaiano, D. A., Ho, C. Y., Chu, V. \& Clifford, A. J. (1980). F. Nutr. 110, 1793.

Simmonds, H. L., Hatfield, P. J., Cameron, J. S., Jones, A. S. \& Cadenhead, A. (1973). Biochem. Pharmacol. 22, 2537.

Sonoda, T. \& Tatibana, M. (1978). Biochim. Biophys. Acta 52 1, 55.

Whittemore, C. T., 'Tullis, J. B. \& Hastie, S. W. (r 978). Br. F. Nutr. 39, 193. 